Signal \& Image Processing : An International Journal (SIPIJ) Vol.6, No.4, August 2015

\title{
SYNTHETIC APERTURE RADAR IMAGES SEGMENTATION USING MINIMUM CROSS- ENTROPY WITH GAMMA DISTRIBUTION
}

\author{
Ali El-Zaart \\ Department of Mathematics and Computer Science, \\ Faculty of Science, Beirut Arab University, Lebanon
}

\begin{abstract}
Computer apparition plays the most important role in human perception, which is limited to only the visual band of the electromagnetic spectrum. The need for Radar imaging systems, to recover some sources that are not within human visual band, is raised. This paper present new algorithm for Synthetic Aperture Radar (SAR) images segmentation based on thresholding technique. Entropy based image thresholding has received sustainable interest in recent years. It is an important concept in the area of image processing. Pal (1996) proposed a cross entropy thresholding method based on Gaussian distribution for bi-modal images. Our method is derived from Pal method that segment images using cross entropy thresholding based on Gamma distribution and can handle bi-modal and multimodal images. Our method is tested using Synthetic Aperture Radar (SAR) images and it gave good results for bi-modal and multimodal images. The results obtained are encouraging.
\end{abstract}

\section{KEYWORDS}

SAR images, Segmentation, Gamma distribution, Cross-Entropy.

\section{INTRODUCTION}

In recent years, SAR imaging has been rapidly gaining fame in applications such as high resolution remote sensing for mapping, surface surveillance, search-and-rescue, mine detection, navigation position location, sensor platform pose control technology, and Automatic Target Recognition (ATR) [12]. For applications such as these, segmentation can play a key role in the subsequent analysis for target detection and recognition. The most important SAR attribute leading to its expanding in opularity is the ability to image large areas of land at fine resolutions and in all weather conditions. A major issue in SAR image is that basic textures are generally affected by multiplicative speckle noise [12]. As we note, in many applications, image segmentation is one of the most difficult and challenging problems. Thresholding is a wellknown and most effective technique for image segmentation according to their simplicity and its speed during processing [3]. It is a technique for converting a grayscale or color image to a binary image based upon a threshold value. The threshold can be chosen manually or by using automated techniques [1]. Threshold methods can be categorized into two groups: global and local methods. Global thresholding technique thresholds the complete image with a single 
Signal \& Image Processing : An International Journal (SIPIJ) Vol.6, No.4, August 2015

threshold value. Local thresholding methods segment the image into a number of sub-images and use a different threshold value for each sub-image. Global thresholding methods are easy to implement and faster due to its less need for computations as proved in [1] and [2]. In [9], Sezgin and Sankur categorize thresholding methods in six groups according to the information they are exploiting. Among these methods, entropy-based methods have drawn the attentions of many researchers. Since it has been proven to be successful and reasonably robust [3]. Minimum Cross Entropy Thresholding (MCET) is a method that adopted in this paper. It selects optimal threshold value that minimizes cross entropy between original and resultant images. $\mathrm{Li}$ and Lee in [2] developed a sequential method to search for optimal threshold value using MCET that based on Gaussian distribution. In [2], $\mathrm{Li}$ and Tam proposed an iterative method that derived for MCET using Gaussian distribution. In [4], Al-Attas and El-Zaart proposed a sequential method for finding optimal threshold value by minimizing Cross Entropy using Gamma distribution to describe data in image. In this paper, we improve the previous works by developing an iterative algorithm for MCET that used for estimating optimal threshold value based on the Gamma distribution. Pal(1996) has derived a new method, which used minimum cross-entropy thresholding method for estimating optimal threshold value based on Gaussian distribution. However, our method process bi-modal and multimodal images, whereas previous method was based on Gamma distribution for the process of bi-modal images.The paper is organized as follows: Section 2 explains the statistical Gamma Distribution. In section 3, we describe image segmentation, section 4 describes image thresholding based on cross Entropy Section 5, presents the Pal cross entropy which is based on Gaussian distribution. Section 6 presents the proposed improvement method of Pal which is based on Gamma distribution. Section 7 presents the experimental results. We conclude in section 8 .

\section{STATISTICAL GAMMA DISTRIBUTION}

Gamma Distribution is a two parameter family of continuous probability distributions in probability theory and statistics. It is a general type of statistical distribution. The probability density function of the Gamma distribution in homogenous area is given by [3][4]:

$f(x, \mu, N)=\frac{2 q}{\mu} \frac{N^{N}}{\tau(N)}\left(\frac{q x}{\mu}\right)^{2 N-1} e^{-N(q x / \mu)^{2}}$

$\quad q=\frac{\tau(N+0.5)}{\tau(N) \sqrt{N}}$, and $\mathrm{x}$ is the intensity of the pixel, $\mu$ is the mean value of the distribution
Where
and $N$ represents the parameter shape of the distribution.

Gamma distribution is having better aptitude than Gaussian as it endow with symmetric and nonsymmetric histograms whereas Gaussian distribution works only with symmetric histograms. For a symmetric histogram, we set $N$ to a high value and to get a histogram skewed to the right set $N$ to a small value (See figure 1). In this figure, if the value of parameter shape $N$ is equal to 1 then the histogram is skewed to the right and if the value of parameter shape $N$ is equal to 10 then the histogram is almost symmetric. 
Signal \& Image Processing : An International Journal (SIPIJ) Vol.6, No.4, August 2015

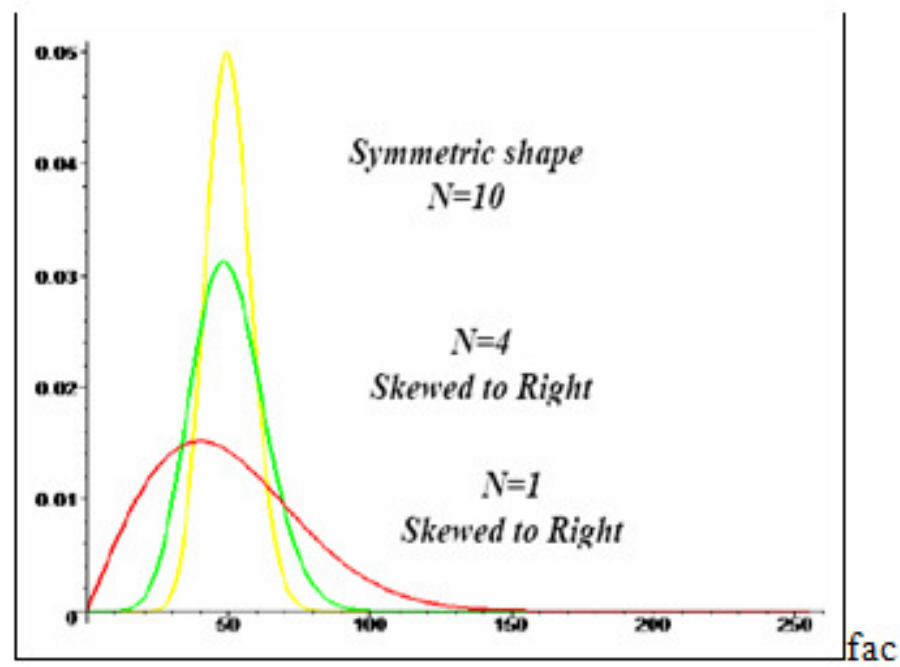

Figure1: Three Gamma distributions with different values of $N$

\section{IMAGE SEGMENTATION}

The goal of segmentation is to simplify the representation of an image into something that is more meaningful and easier to analyze. Image thresholding, one of the most important techniques for image segmentation, the objective of this operation is that the objects and background are separated into non-overlapping sets $[13,14,15,17,18,19]$. Image thresholding is necessary step in many image analysis applications. Image thresholding can be handled globally by determining a single threshold value for an entire image or locally apply different threshold value in different spatial regions $[6,13]$. Thresholding techniques can be divided into bi-level and multilevel category depending on number of image segments. Image Thresholding classifies pixels into two categories - Those to which some property measured from the image falls below a threshold, and those at which the property equals or exceeds a threshold. Thresholding creates a binary image (Binarization) using the following formula:

$$
s(x, y)=\left\{\begin{array}{l}
1-----I(x, y)>T \\
0-----I(x, y) \leq T
\end{array}\right.
$$

Where $\mathrm{s}(\mathrm{x}, \mathrm{y})$ is a segmented/thresholded image, $\mathrm{I}(\mathrm{x}, \mathrm{y})$ is the original image, $\mathrm{T}$ is the Threshold [1]. Many different methods have been accessible to get the threshold valued. Surveys were conducted and have been proposed to binarize an image [7]. Thresholding methods have been classified into six categories [ ref]. Entropy based Method is wieldy used in image thesholding. In next section, we will explain the entropy based thresholding.

\section{IMAGE THRESHOLDING BASED ON ENTROPY}

Entropy is a measure of the number of random ways in which a system may be arranged. Entropy measure the information content in probability distribution. It separates the information into two regions. Entropy based thresholding considers an image histogram as a probability distribution. There are two techniques developed for entropy based thresholding -the local entropy (LE) and joint entropy (JE). Entropy based thresholding usually considers and image histogram as a probability distribution, and then selects an optimal threshold value [8]. Many entropy based thresholding methods have been proposed in the literature. These methods are classified into 
Signal \& Image Processing : An International Journal (SIPIJ) Vol.6, No.4, August 2015

three groups: entropic thresholding, fuzzy entropic thresholding and cross entropic thresholding. Entropic thresholding "considers the image foreground and background as two different signal sources, so that when the sum of the two class entropies reaches its maximum, the image is said to be optimally thresholded" [9]. Fuzzy entropic thresholding "considers the fuzzy memberships as indicators of how strongly a grey value belongs to the background or to the foreground" [9]. Cross entropic thresholding "considers the thresholding as the minimization of an information theoretic distance" [9]. This paper is focused on the cross entropy thesholding method. Many algorithms have been proposed for cross entropy thresholding, among them are Li and Lee (1993) have introduced the minimum cross entropy thresholding algorithm that selects the threshold, which minimizes the cross entropy between the segmented image and the original image [10]. Li and Lee have proposed a method that is based on Gaussian distribution in sequential manner [3]. They used the kullback's information theoretic distance $D$ between two probability distributions [3], $D$ is also known as directed divergence or cross entropy. Let $\mathrm{I}=$ $\left\{f_{1}, f_{2}, \ldots . . f_{n}\right\}$ be the distribution of original image and $\mathrm{It}=\left\{g_{1}, g_{2}, \ldots . g_{n}\right\}$ the distribution of thresholded image then the cross entropy is defined as follows:

$$
D\left(I, I_{t}\right)=\sum_{i=0}^{n} f_{i} \log \left(f_{i} / g_{i}\right)
$$

$\mathrm{D}(\mathrm{I}, \mathrm{It})$ is non-symmetric.

Li and Tam (1998) developed an iterative method derived from Li and Lee [2] which derived the threshold value from minimizing the cross entropy using Gaussian distribution [2]. Al-Attas and El-Zaart (2006) have proposed a sequential method derived from $\mathrm{Li}$ and Lee [10] and $\mathrm{Li}$ and Tam [2] to find optimal threshold value by minimizing cross entropy using Gamma distribution[4]. The method resulted good outcome in medical images. Al-Osaimi and El-Zaart (2008) developed a new iterative algorithm derived from $\mathrm{Li}$ and Lee [10] and $\mathrm{Li}$ and Tam [2] to find optimal threshold value by minimizing cross entropy using Gamma distribution [5].

$$
D\left(I, I_{t}\right)=\sum_{i=0}^{n} f_{i} \log \left(f_{i} / g_{i}\right)+\sum_{i=0}^{n} g_{i} \log \left(g_{i} / f_{i}\right)
$$

Brink and Pendock in [16] redefine the information theoretic distance as:

$$
D\left(I, I_{t}\right)=\sum_{i=1}^{L} f(i) \log \frac{f(i)}{g(i)}+\sum_{i=1}^{L} g(i) \log \frac{g(i)}{f(i)}
$$

El-Zaart worked on Brink cross entropy which is an improvement of Lin and Lee cross entropy $[13,14,15,16]$. Pal[11] is developed a cross entropy method that is more general than Li and Brink. Next section we will explain the Pal method.

\section{PAL Thresholding MeThOd BASED ON GAUSSIAN Distribution}

A cross entropy thresholding method where image histogram is modeled by a mixture of Poisson distributions was proposed by Pal [11]. In general any thresholding method segments the image into object and background regions based on the threshold t. The gray level values of object is defined from [0-t] and the gray level values of background is defined from $[\mathrm{t}+1, \mathrm{~L}]$, where $\mathrm{L}$ is the possible number of gray levels. Then the distribution of gray level in the object and background regions can be defined as: 
Signal \& Image Processing : An International Journal (SIPIJ) Vol.6, No.4, August 2015

$f_{O}=\left\{f_{1}^{O}, f_{2}^{O}, \ldots \ldots \ldots . ., f_{t}^{O}\right\}$ and $f_{B}=\left\{f_{t+1}^{B}, f_{t+2}^{B}, \ldots \ldots \ldots ., f_{L}^{B}\right\}$,

Where: $f_{i}^{o}=\frac{h_{i}}{\text { sum }} i=1,2,3, \ldots \ldots \ldots . t$ and $f_{i}{ }^{B}=\frac{h_{i}}{M \times N-\operatorname{sum}}, i=t+1, t+2, \ldots \ldots \ldots . . L$

Where sum $=\sum_{i=1}^{t} h_{i}$

Note that: $\sum_{i=1}^{t} f_{i}{ }^{o}=1$ and $\sum_{i=t+1}^{L} f_{i}{ }^{B}=1, \mathrm{M} \times \mathrm{N}$ is the width and the length of the image, and $h_{i}$ is the histogram of gray level $\mathrm{i}$.

We consider two Poisson probability distributions $G_{O}$ and $G_{B}$ for object and background respectively,

$$
\begin{aligned}
& G_{O}=\left\{g_{1}^{O}, g_{2}^{O}, \ldots \ldots g_{t}^{O}\right\}, G_{B}=\left\{g_{t+1}^{B}, g_{t+2}^{B}, \ldots \ldots . g_{L}^{B}\right\} \\
& g_{i}^{O}=\frac{e^{-\mu_{O}} \mu_{o}^{i}}{i !} i=1,2, \ldots \ldots \ldots t \quad \text { and } \quad g_{i}^{B}=\frac{e^{-\mu_{B}} \mu_{B}^{i}}{i !} i=t+1, t+2, \ldots \ldots \ldots L
\end{aligned}
$$

Where $\mu_{O}$ and $\mu_{B}$ are the mean value of object and background regions and they estimated using Gaussian distribution as follows:

$\mu_{O}=\left(\sum_{i=1}^{t} i h_{i}\right) / \sum_{i=1}^{t} h_{i}$ and $\mu_{B}=\left(\sum_{i=t+1}^{L} i h_{i}\right) / \sum_{i=t+1}^{L} h_{i}$

The cross entropy for the object and background regions are :

$$
\begin{aligned}
& D_{O}(t)=D_{t}\left(f_{O}, G_{O}\right)=\sum_{i=1}^{t} f_{i}^{O} \log \left(f_{i}^{O} / g_{i}^{o}\right)+\sum_{i=1}^{t} g_{i}^{o} \log \left(g_{i}^{O} / f_{i}^{O}\right) \\
& D_{B}(t)=D_{t}\left(f_{B}, G_{B}\right)=\sum_{i=t+1}^{L} f_{i}^{B} \log \left(f_{i}^{B} / g_{i}^{B}\right)+\sum_{i=t+1}^{L} g_{i}^{B} \log \left(g_{i}^{B} / f_{i}^{B}\right)
\end{aligned}
$$

The total cross entropy is : $D(t)=D_{o}(t)+D_{B}(t)$

\section{IMPROVEMENT OF PAL METHOD}

Pal (1996) developed a thresholding method for images containing only two classes (bi-modal thresholding) by assuming that the data in image is modelled by Gaussian distribution. There are two problems in the Pal method: (i) if the image contains more than two classes that needs more than one threshold and (ii) if the data in image is not symmetric which means that Gaussian 
Signal \& Image Processing : An International Journal (SIPIJ) Vol.6, No.4, August 2015

distribution is not suitable. In our improvement of Pal method we solved these two problems by first extending the method to multimodal thresholding and second by using a more general distribution than Gaussian. We used Gamma distribution [4,12] which can model symmetric and non-symmetric data. Thus, the image is composed of two Gamma distributions; the first is for the object region and the second for the background region. The estimated means for these two regions are [4]:

$\mu_{O}(t)=\sqrt{\left(\sum_{i=0}^{t} h(i) i^{2} q^{2}\right) / \sum_{i=0}^{t} h(i)} \quad$ and $\quad \mu_{B}(t)=\sqrt{\left(\sum_{i=t+1}^{L} h(i) i^{2} q^{2}\right) / \sum_{i=t+1}^{L} h(i)}$

Where h(i) is the histogram defined on the gray level $[0, \mathrm{~L}]$ and $q=\frac{\tau(N+0.5)}{\tau(N) \sqrt{N}}$.

The improvement of $\mathrm{Pal}$ cross entropy thresholding algorithm using Gamma distribution is as follows:

$$
\begin{aligned}
& D(t)=\sum_{i=1}^{t} f_{i}^{O} \log \left(f_{i}^{O} / g_{i}^{O}\right)+\sum_{i=1}^{t} f_{i}^{O} \log \left(g_{i}^{O} / f_{i}^{O}\right)+ \\
& \sum_{i=t+1}^{L} f_{i}^{B} \log \left(f_{i}^{B} / g_{i}^{B}\right)+\sum_{i=t+1}^{L} g_{i}^{B} \log \left(g_{i}^{B} / f_{i}^{B}\right)
\end{aligned}
$$

The optimal value of $\mathrm{t}^{*}$ is the value which minimizes the objective function $D(t)$ [11].

\subsection{Improvement Bi-modal Algorithm of Pal:}

\section{Begin}

Set $\min =\max$ value of Int as initial value for $\mathrm{t}=1 . .255$

Begin

Compute $\mu_{O}$ using Gamma distribution

Compute $\mu_{B}$ using Gamma distribution

Compute $\quad f_{i}^{o}, i=0, \ldots \ldots ., t$

Compute $f_{i}^{B}, i=t+1, \ldots, 255$

Compute $g_{i}^{o}, i=0, \ldots, t$

$$
g_{i}^{B}, i=t+1, \ldots, 255
$$

Compute $D(t)$ using equation

if $(\min >D(T))$

Begin

$\min =D(t)$

threshold $=t$

end

end

end 


\subsection{New Multimodal Thresholding Algorithm}

We extended the bi-model thresholding method to multimodal using Gamma distribution. Assume that we have $m$ classes of pixels in an image. Then we have a threshold vector of $\mathrm{m}-1$ thresholds $\mathrm{T}=\left\{t_{1}, t_{2}, t_{3} \ldots \ldots . . t_{m-1}\right\}$, where $t_{0}<t_{1}<t_{2}<t_{3}<\ldots \ldots . . t_{m-1}<t_{m}, \mathrm{t} 0=0$ and $t_{m}=255$. The multimodal cross-entropy is defined as follows:

$$
\begin{aligned}
& D(t)=\left(\sum_{i=t_{0}}^{t_{1}} f_{i}^{O} \log \left(\frac{f_{i}^{O}}{g_{i}^{O}}\right)+\sum_{i=t_{0}}^{t_{1}} g_{i}^{O} \log \left(\frac{g_{i}^{O}}{f_{i}^{O}}\right)\right)+\left(\sum_{i=t_{1}+1}^{t_{2}} f_{i}{ }^{B} \log \left(\frac{f_{i}^{B}}{g_{i}^{B}}\right)+\sum_{i=t_{1}+1}^{t_{2}} g_{i}^{B} \log \left(\frac{g_{i}^{B}}{f_{i}^{B}}\right)\right) \\
& +\ldots \ldots \ldots \ldots \ldots+\left(\sum_{i=t_{m-1} 1}^{L-1} f_{i}^{B} \log \left(\frac{f_{i}^{B}}{g_{i}^{B}}\right)+\sum_{i=t_{m-1}}^{L-1} g_{i}^{B} \log \left(\frac{g_{i}^{B}}{f_{i}{ }^{B}}\right)\right)
\end{aligned}
$$

The mean value of $\mathrm{k}^{\text {th }}$ class, will be estimated using Gamma distribution:

$$
\mu_{K}(t)=\sqrt{\left(\sum_{i=t_{k-1}}^{t_{k}} h(i) i^{2} q^{2}\right) / \sum_{i=t_{k-1}}^{t_{k}} h(i)}
$$

The proposed new Multimodal algorithm is as follows:

1-Initial values of thresholds can be estimated using $k$-mean algorithm

$$
T 0=\left\{t_{1}, t_{2}, t_{3} \ldots \ldots . . t_{m-1}\right\}, t_{0}=0 \text { and } t_{m}=255
$$

2-On each two consecutive modes in the multimodel histogram, apply bi-modal thresholding algorithm, in order to obtain the new value of threshold $T_{\text {new }}(k)$. These two modes starting from $t_{k-1}$ and ending at $t_{k}$. For $k=1,2 \ldots m-1$.

3-We compare the two threshold vectors $T_{0}$ and $T_{n e w}$

$$
\text { If }\left|T_{0}-T_{\text {new }}\right|<\varepsilon \text { then optimal threshold values } T^{*}=\left\{t_{1}^{*}, t_{2}^{*}, t_{3}^{*} \ldots \ldots . . . t_{m-1}^{*}\right\} \text { are reached }
$$

else Assign TO $\longleftarrow T_{\text {new }}$ and go to step 2

\section{EXPERIMENTAL RESULTS}

The improvement bi-modal Pal method is implemented using Gamma distribution and applied on several real SAR images, and then the . Multimodal thresholding is applied on multimodal image.

\subsection{SAR Images with Two classes}

In this section, we will apply the improved bimodal thresholding method on three real SAR images, where the parameter $\mathrm{N}$ is given by the user.

- Figure 2a presented a real SAR image, we applied the bimodal algorithm on this image with value of $\mathrm{N}=10$. The segmented image presented in figure $2 \mathrm{~b}$ with estimated threshold $\mathrm{T}=30$. Figure $2 \mathrm{c}$, presented the histogram of the real SAR image with the estimated threshold in green line. 
Signal \& Image Processing : An International Journal (SIPIJ) Vol.6, No.4, August 2015

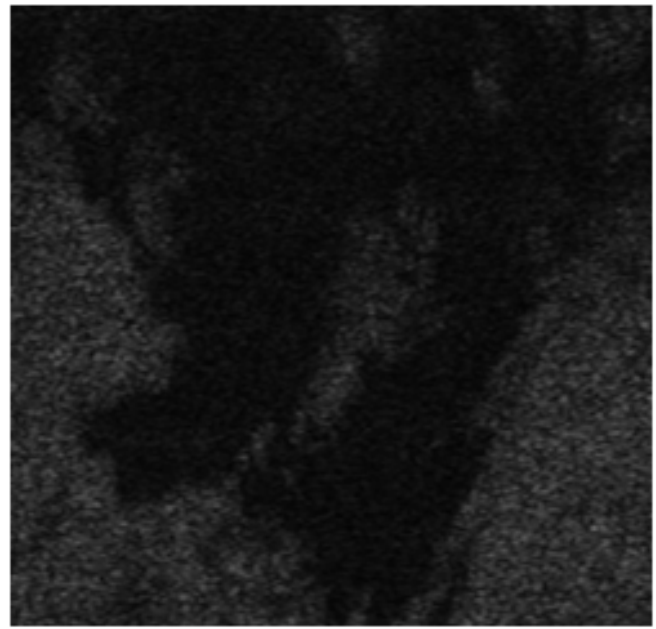

(a)

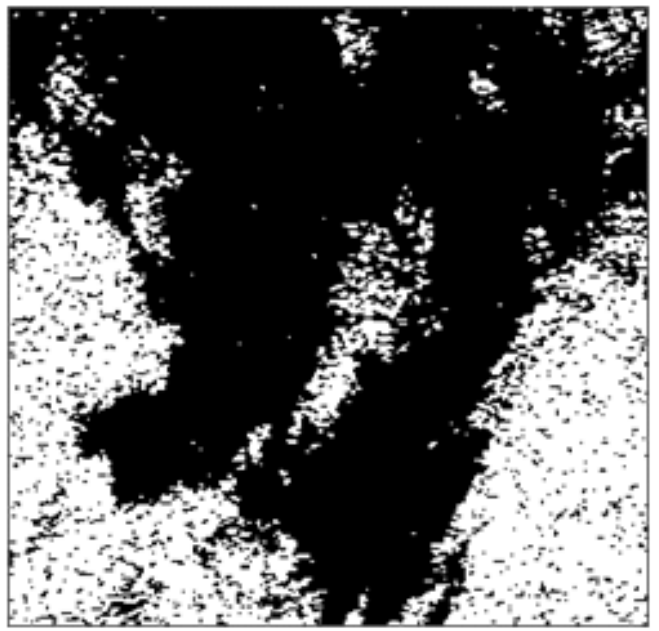

(b)

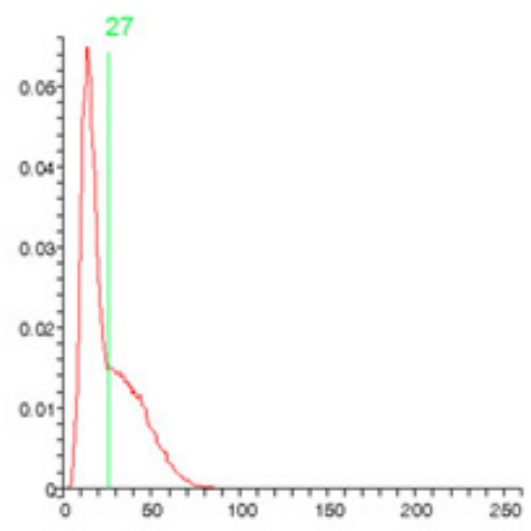

(c)

Figure 2: (a) Real SAR image. (b) Segmented image with $\mathrm{N}=10$ and $\mathrm{T}=30$.

(c) Image histogram with estimated threshold.

- Figure 3a presented a real SAR image, we applied the bimodal algorithm on this image with value of $\mathrm{N}=8$. The segmented image presented in figure $3 \mathrm{~b}$ with estimated threshold $\mathrm{T}=29$. Figure $3 \mathrm{c}$, presented the histogram of the real SAR image with the estimated threshold in green line. 
Signal \& Image Processing : An International Journal (SIPIJ) Vol.6, No.4, August 2015

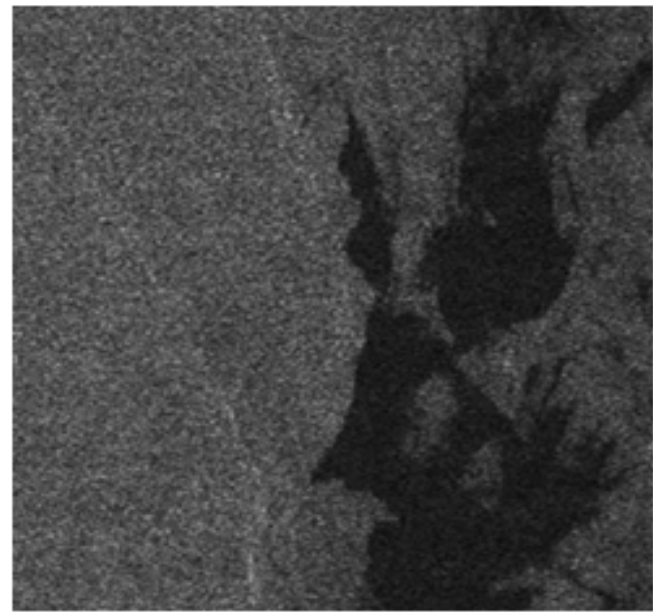

(a)

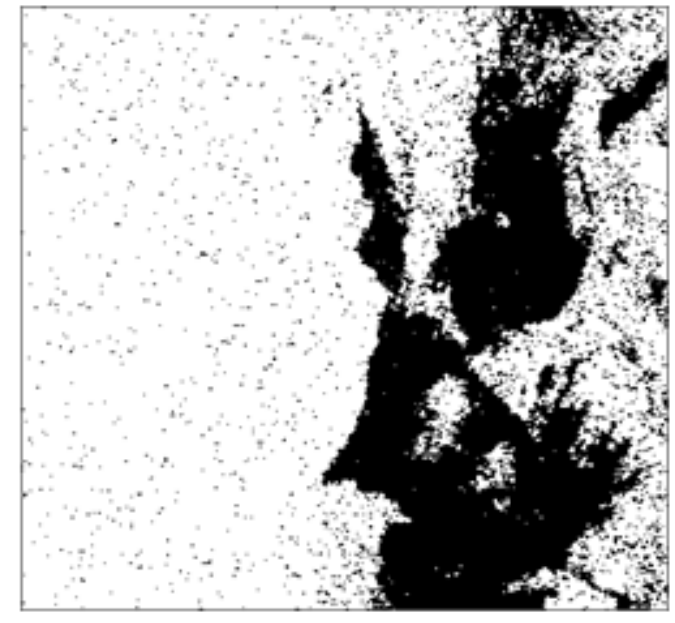

(b)

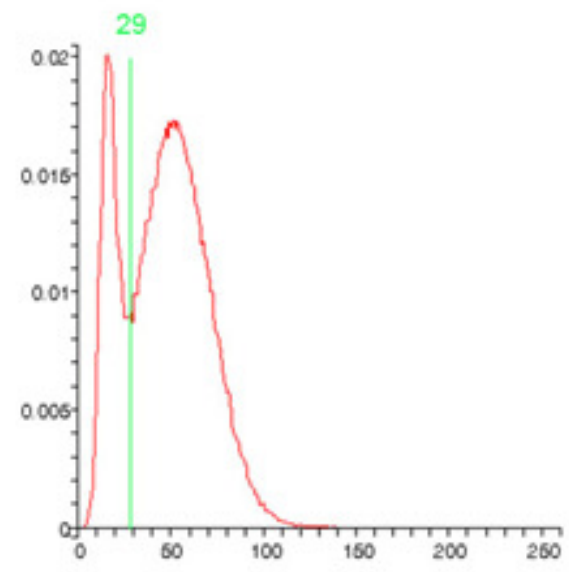

(c)

Figure3: (a) Real SAR image. (b) Segmented image with $\mathrm{N}=8$ and $\mathrm{t}=29$.

(c) Image histogram with estimated threshold

- Figure 4a presented a real SAR image, we applied the bimodal algorithm on this image with value of $\mathrm{N}=12$. The segmented image presented in figure $4 \mathrm{~b}$ with estimated threshold $T=27$. Figure $4 c$, presented the histogram of the real SAR image with the estimated threshold in green line. 
Signal \& Image Processing : An International Journal (SIPIJ) Vol.6, No.4, August 2015

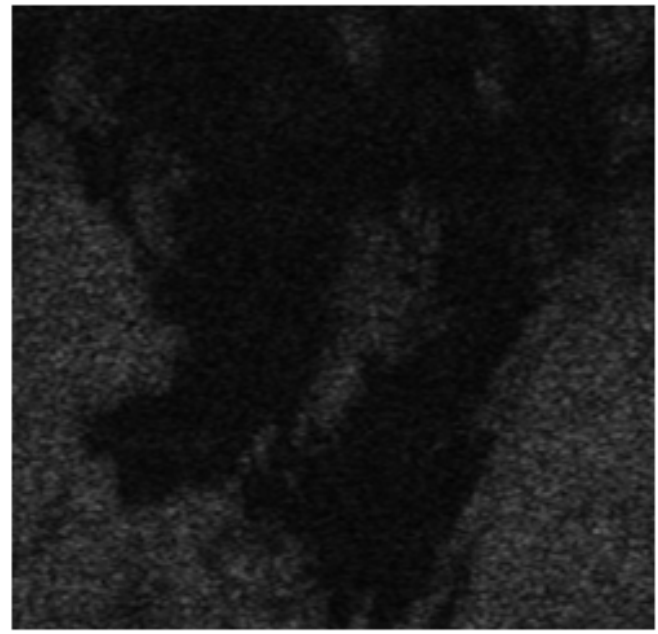

(a)

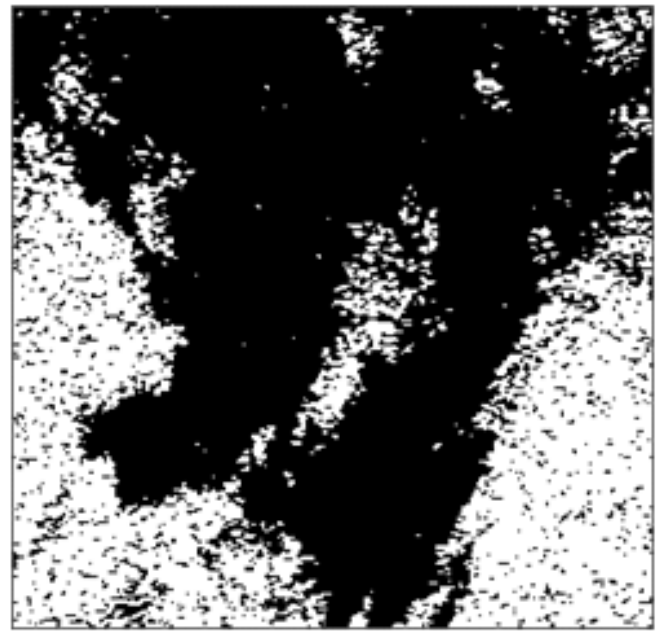

(b)

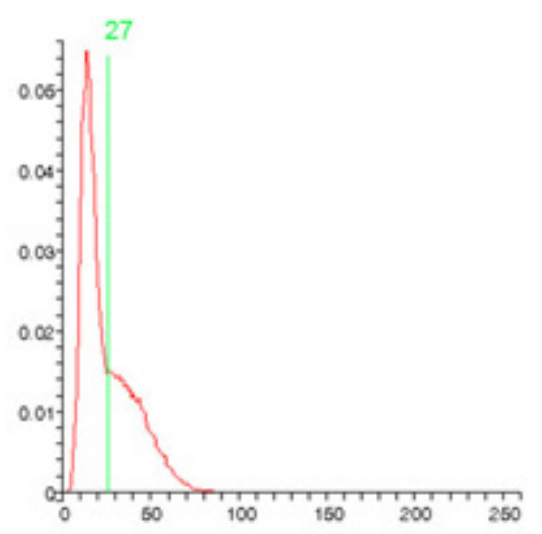

(c)

Figure 4: (a) Real SAR image. (b) Segmented image with $\mathrm{N}=12$ and $\mathrm{T}=27$.

(c) Image histogram with estimated threshold

\subsection{Real SAR Image with Three Classes}

Figure 5a presented a real SAR image with three classes of pixels, the proposed multimodal thresholding method is applied to this image with $\mathrm{N}=12$. The estimated thresholds are $\mathrm{T} 1=14$ and $\mathrm{T} 2=42$. The segmented image is presented in figure $5 \mathrm{~b}$. Figure $5 \mathrm{c}$ presented the histogram of real SAR image with two estimated thresholds. 
Signal \& Image Processing : An International Journal (SIPIJ) Vol.6, No.4, August 2015

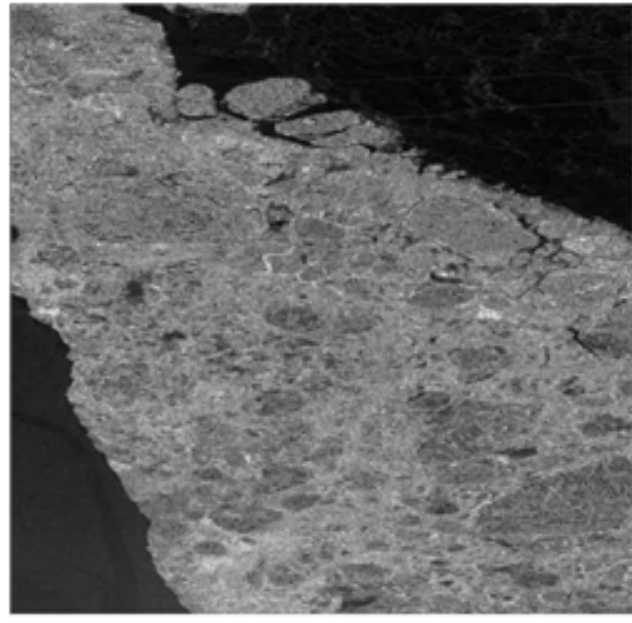

(a)

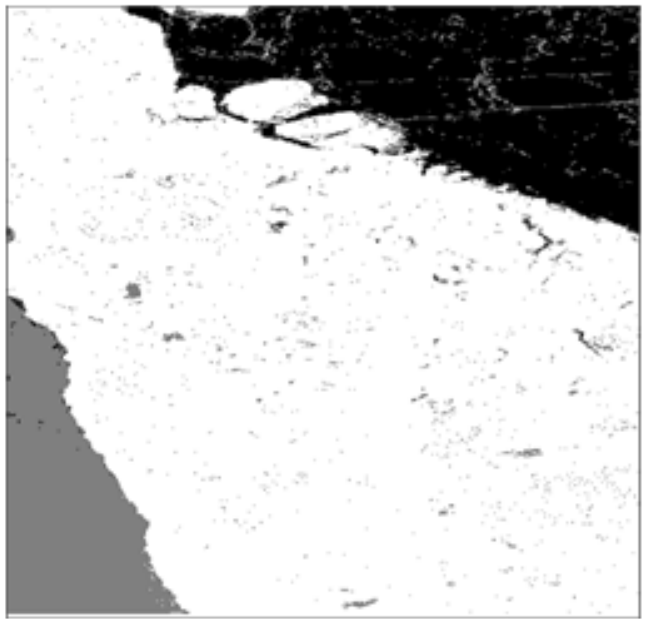

(b)

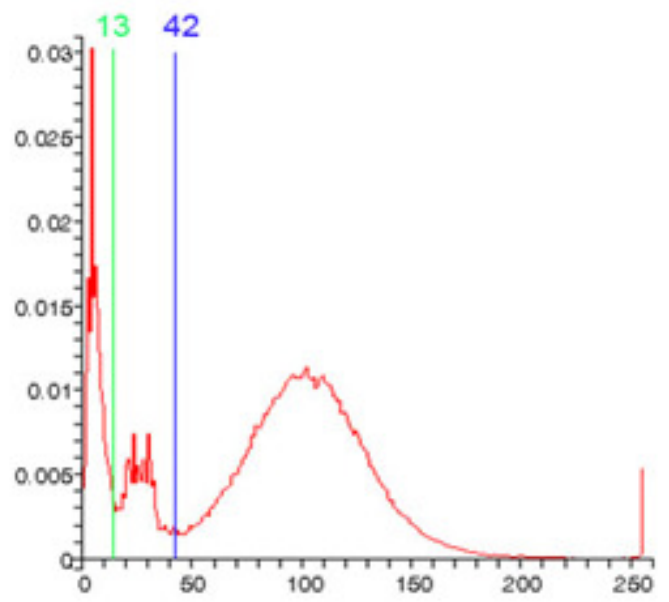

(c)

Figure 5: (a) Real SAR image. (b) Segmented image with $N=12, T 1=13$ and $T 2=42$. (c) Image histogram two estimated thresholds

\section{CONCLUSION AND FUTURE WORK}

In this paper, we proposed am improvement of the Pal cross entropy thresholding. Pal (1996) developed a thresholding method for images containing only two classes (bi-modal thresholding) by assuming that the data in image is modelled by Gaussian distribution. In our improvement of Pal method we extended his work for multimodal thresholding and using a more general distribution than Gaussian. We used Gamma distribution which can model symmetric and nonsymmetric data. Thus, the image is composed of two Gamma distributions; the first is for the object region and the second for the background region. The experimental results showed that the improvement method segmented well the SAR images having two and three classes. The estimated threshold is done in sequential manner, as future work we will develop method that can find the optimal threshold using iterative method. 
Signal \& Image Processing : An International Journal (SIPIJ) Vol.6, No.4, August 2015

\section{REFERENCES}

[1] Rafael C.gonzalez, Richard E.Woods, "Digital Image Processing," Prentice Hall, New York, $3^{\text {rd }}$ Edition, 2008

[2] C.H.Li and P K S Tam, "An iterative algorithm for minimum cross entropy thresholding", Pattern Recognition Vol 19 (1998), pp 771-776

[3] ŞENGÜR, I. TÜRKOĞLU and M. İNCE, "A Comparative Study On Entropic Thresholding Methods" Journal of Electrical \& Electronics Engineering, vol.6, no. 2, 2006, pp. 183-188.

[4] R. Al-Attas, and A. El-Zaart, "Thresholding of Medical Images Using Minimum Cross Entropy", kuala lumpur international conference on biomedical engineering, kuala lumpur, Malaysia, 2006, pp. 312-315.

[5] G. Al-Osaimi and A. El Zaart, "Minimum Cross Entropy Thresholding for SAR Images", 3rd IEEE International Conference on Information \& Communication Technologies: From Theory to Applications ICTTA. Syria, April 2008

[6] Wonho Oh, W. Brent Lindquist,"Image Thresholding by Indicator Kriging", IEEE on Pattern Analysis and Machine Intelligence, $\mathrm{Vol} 20$

[7] B. Sankura,, and M. Sezgin, "Image Thresholding Techniques: a Survey over Categories", Pattern Recognition, 2001

[8] C. Chang,Y. Du, J. Wang, S. Guo and P. Thouin, "Survey and Comparative Analysis of Entropy and Relative Entropy Thresholding Techniques", IEEE Proc.-Vis. Image Signal Process, vol. 153, no. 6, December 2006, pp. 837-850.

[9] M. Sezgin, and B. Sankur, "Survey Over Image Thresholding Techniques And Quantitative Performance Evaluation", Journal of Electronic Imaging, vol. 13, no. 1, January 2004, pp. 146-165

[10] C. Li, and C. Lee, "Minimum Cross Entropy Thresholding", Pattern Recognition-Elsevier, vol. 26, 1993, pp. 617-625.

[11] N.R. Pal, "On Minimum Cross-Entropy Thresholding", Pattern Recognition Journal-Elsevier, vol.29, no. 4,1996 , pp. $575-580$.

[12] A. El Zaart, D. Ziou, S. Wang, and O. Jiang, "Segmentation of SAR images," Pattern Recognition Journal, vol. 35, no. 3, March 2002, pp. 713-724.

[13] Roula Alayli and Ali El-Zaart, "An Iterative Mammographic Image Thresholding Algorithm For Breast Cancer Detection", submitted to The 2012 International Arab Conference on Information Technology (ACIT'2012). Lebanon. December 201

[14] Ali Ghosn and Ali El-Zaart, Skin Images Segmentation Using Between Class Variance with Gamma Distribution International Science Conference (LAAS20), Hadath, Lebanon, March 2014.

[15] Moumena Al-Bayati and Ali El-Zaart, Automatic Thresholding Techniques for SAR Images", International Concefrence in Soft Computing (2013). Dubai, 18-19 May 2013.

[16] Ali El-Zaart and Ali Ghosn, SAR images Thresholding For Oil Spill Detection ”, The 2nd International Electronics, Communications, and Photonics Confefence.Riyadh, Saudi Arabia, 2013.

[17] Fari Muhammad Abubakar, A study of Region-Based and contour-Based images segmentation, Signal \& Image Processing : An International Journal (SIPIJ) Vol.3, No.6, December 2012.

[18] Nuseiba M. Altarawneh, Suhuai Luo, Brian Regan, Changming Sun, Fucang Jia, Global Threshold and Region-Based active contour for accurate image segmentation. Signal \& Image Processing: An International Journal (SIPIJ) Vol.5, No.3, June 2014.

[19] Mokhtar M. Hasan, , Pramod K. Mishra, Novel Algorithm for Skin Color Based Segmentation Using Mixture of GMMS, Signal \& Image Processing : An International Journal (SIPIJ) Vol.4, No.4, August 2013. 
Signal \& Image Processing : An International Journal (SIPIJ) Vol.6, No.4, August 2015

\section{AUTHOR}

Ali El-Zaart was a senior software developer at Department of Research and Development, Semiconductor Insight, Ottawa, Canada during 2000-2001. From 2001 to 2004, he was an assistant professor at the Department of Biomedical Technology, College of Applied Medical Sciences. From 2004-2011, he was an assistant professor and then associate professor at the Department of Computer Science, College of computer and information Sciences. Since 2011 he is an associate professor at the department of Mathematics and computer science, Faculty of Science, Beirut Arab University, Lebanon. He published more than 110 articles and proceedings in the areas of image processing, remote sensing, and computer vision. He received a B.Sc. in computer science from the University of Lebanon; Beirut, Lebanon in 1990, M.Sc. degree in computer science (image processing) from the University of Sherbrooke, Sherbrooke, Canada in 1996, and $\mathrm{Ph} . \mathrm{D}$. degree in computer science (image processing) from the University of Sherbrooke, Sherbrooke, Canada in 2000. His research interests include image processing, pattern recognition, remote sensing, and computer vision, Data Mining, e-government, smart cities. 